\title{
Autophagy induction contributes to the resistance to methotrexate treatment in rheumatoid arthritis fibroblast-like synovial cells through high mobility group box chromosomal protein 1
}

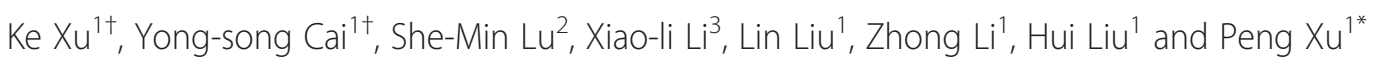

\begin{abstract}
Background: Rheumatoid arthritis fibroblast-like synovial cells (RA-FLS) show resistance to methotrexate (MTX) treatment. To better understand the mechanisms of this resistance, RA-FLS and osteoarthritis fibroblast-like synovial cells (OA-FLS) were isolated and exposed to MTX. We analyzed the autophagy induced by MTX in vitro and its relationship to apoptosis.

Methods: Cell viability was evaluated using a 3-(4,5-dimethylthiazol-2-yl)-2,5-diphenyltetrazolium bromide assay, and apoptosis was detected by flow cytometry and Western blot analysis. Autophagy was determined by transmission electron microscopy as well as Western blot analysis. The expression levels of Beclin-1, LC3, Akt, p-Akt, mammalian target of rapamycin (mTOR), p-mTOR, high mobility group box chromosomal protein 1 (HMGB1), and an $85 \mathrm{kDa}$ caspase cleaved fragment of poly(ADP-ribose) polymerase were measured by Western blotting.

Results: MTX-induced apoptosis was increased in OA-FLS compared with RA-FLS. However, MTX stimulated the autophagy response in RA-FLS by inducing autophagosome formation, but not in OA-FLS. In RA-FLS, transfection with Beclin-1 small interfering RNA inhibited autophagy and increased susceptibility to MTX, which induces cell death. MTX upregulated autophagy through its ability to enhance the expression of HMGB1 and Beclin-1 rather than through the Akt/mTOR pathway.
\end{abstract}

Conclusions: Autophagy induction contributes to resistance to MTX treatment in fibroblasts from patients with rheumatoid arthritis.

Keywords: Rheumatoid arthritis, Fibroblast-like synovial cells, Methotrexate, Autophagy, Apoptosis

\section{Background}

With a high prevalence and an associated level of disability, rheumatoid arthritis (RA) is the most common serious autoimmune disease in several parts of the world. To date, it is widely accepted that both the inflammatory and destructive features of RA are driven by synovitis, characterized not only by increases in the

\footnotetext{
* Correspondence: sousou369@163.com

${ }^{\dagger}$ Equal contributors

${ }^{1}$ Department of Joint Surgery, Xi'an Hong Hui Hospital, Xi'an Jiaotong University Health Science Center, Xi'an 710054, Shaanxi Province, China

Full list of author information is available at the end of the article
}

number and activity of lymphocytes and macrophages but also by the numbers of resident mesenchymal cells, known as fibroblast-like synovial cells (FLS) [1]. Located in the synovial lining, FLS derived from RA appear to change their phenotype to become hyperplastic and invasive, like tumor cells [2]. Accumulating evidence indicates that the transformation of rheumatoid arthritis fibroblast-like synovial cells (RA-FLS) occurs as a result of both defective apoptosis and excessive proliferation.

The folic acid antagonist methotrexate (MTX), which is a potent, competitive inhibitor of dihydrofolate reductase, is the most widely used of the disease-modifying 
antirheumatic drugs (DMARDs) in the treatment of RA $[3,4]$. Compared with the $5000 \mathrm{mg} /$ week dosage used in the treatment of malignancy, once-weekly administration of MTX at 7.5-25 mg produces optimal clinical outcomes in RA [5]. According to previous studies, apart from the anti-inflammatory effects, MTX suppresses the proliferation of lymphocytes and macrophages but has no effect on spontaneous proliferation of RA-FLS $[6,7]$. Its promotion of RA-FLS apoptosis is rather limited [8-11].

Autophagy is a survival strategy employed by cells undergoing nutrient deprivation or other stresses. Increasing evidence indicates that autophagy protects various tumor cells from apoptosis induced by chemotherapy drugs, both in vivo and in vitro [12-14]. Nevertheless, extensive or persistent autophagy also produces cell death. Thus, autophagy often serves as an adapter between cell death and survival [15]. Compared with osteoarthritis (OA), both enhanced autophagy in RA synovial tissues and increased induction of autophagy in RA-FLS were recently described [16, 17]. Autophagy exerted protective effects in these studies. This raises a question about the role of autophagy in the process of MTX treatment of RA-FLS.

On the basis of the present study, we report that MTX induced protective autophagy in RA-FLS and that inhibition of autophagy enhanced MTX-induced apoptosis of RA-FLS.

\section{Methods}

\section{Human tissue collection}

Synovial tissue specimens were obtained from seven patients with $\mathrm{OA}$ and seven patients with RA during joint replacement surgery (Department of Joint Surgery, Hong Hui Hospital, Xi'an, China). This study was approved by the human research ethics committee of the Xi'an Hong Hui Hospital, and all patients fulfilled the American College of Rheumatology criteria for classification of RA. All patients provided informed consent. Clinical data, laboratory examination results, and patient medications are summarized in Table 1.

\section{Reagents and antibodies}

MTX and antibodies against microtubule-associated protein 1 light chain 3 (anti-LC3) were purchased from Sigma-Aldrich (St. Louis, MO, USA). Anti- $\beta$-actin antibodies were purchased from Biosen (Beijing, China). High mobility group box chromosomal protein 1 (HMGB1), anti-poly(ADP-ribose) polymerase (PARP), anti-phosphorylated mammalian target of rapamycin (anti-p-mTOR), and anti-mTOR antibodies were purchased from Abcam (Cambridge, UK). Anti-Beclin-1, anti-Akt, and anti-phosphorylated Akt (anti-phosphoAkt; Ser473) antibodies were purchased from Cell Signaling Technology (Danvers, MA, USA).
Table 1 Patient characteristics

\begin{tabular}{lll}
\hline Patient characteristics & OA & RA \\
\hline Total number of patients & 7 & 7 \\
Age, $^{\text {a }} \mathrm{yr}$ & $68(58-70)$ & $61(55-75)$ \\
Sex, $n$, female/male & $2 / 5$ & $5 / 2$ \\
$\mathrm{CRP}^{\mathrm{b}} \mathrm{mg} / \mathrm{L}$ & $2.72(2.25)$ & $17.75(26.52)$ \\
$\mathrm{RF}^{\mathrm{b}}{ }^{\mathrm{V}} \mathrm{U} / \mathrm{ml}$ & $9.16(2.90)$ & $102.31(70.07)$ \\
Anti-CCP-positive, $n$ & $0 / 7$ & $6 / 7$ \\
ESR, $^{\mathrm{b}} \mathrm{mm} / \mathrm{h}$ & $12.43(6.55)$ & $39(15.12)$ \\
Medications, number of patients & & \\
NSAIDs & 7 & 7 \\
Steroids & 3 & 1 \\
TWP & 0 & 3 \\
MTX & 0 & 4 \\
\hline
\end{tabular}

CRP C-reactive protein, ESR erythrocyte sedimentation rate, MTX methotrexate, $R F$ rheumatoid factor, $C C P$ cyclic citrullinated peptide, NSAID nonsteroidal anti-inflammatory drug, TWP Tripterygium wilfordii polyglycoside ${ }^{a}$ Median (range)

${ }^{\mathrm{b}}$ Mean (standard error of the mean)

\section{Cell culture and treatment}

Synovial fibroblasts were isolated from synovial tissue specimens obtained from patients with RA and patients with OA. Cells were cultured as described elsewhere [18] and used between passages 3 and 8 for all experiments. The concentrations of MTX used in the different experiments ranged from $0.01 \mu \mathrm{M}$ to $1 \mu \mathrm{M}$, and the culture periods ranged from $24 \mathrm{~h}$ to $96 \mathrm{~h}$ of continuous exposure to MTX. On the basis of pharmacokinetic analysis, the ingestion of a 20-mg tablet of MTX yields plasma MTX concentrations of approximately $0.5 \mu \mathrm{M}$ after $1 \mathrm{~h}$ and of approximately $0.1 \mu \mathrm{M}$ after $10 \mathrm{~h}$ [19]. Controls were treated with matched amounts of dimethyl sulfoxide (DMSO); $0.1 \mu \mathrm{M}$ of bafilomycin A1 (Sigma-Aldrich), which inhibits the fusion of autophagosomes with lysosomes, was added to cell cultures for the last $4 \mathrm{~h}$ of treatment.

\section{Cell viability assay}

Cell viability was measured using a 3-(4,5-dimethylthiazol2-yl)-2,5-diphenyltetrazolium bromide (MTT) assay. The cells were seeded at $5 \times 10^{4}$ cells/well in 96-well plates, incubated overnight, and then exposed to the indicated concentrations of MTX for the indicated times. Thereafter, $20 \mu \mathrm{l}$ of MTT solution $(5 \mathrm{mg} / \mathrm{ml})$ was added to each well, and the cells were incubated for another $4 \mathrm{~h}$ at $37{ }^{\circ} \mathrm{C}$. After removal of the culture medium, the cells were lysed in $200 \mu \mathrm{l}$ of DMSO, and the optical density (OD) was measured at $570 \mathrm{~nm}$ with a microplate reader (Thermo Fisher Scientific, Waltham, MA, USA). The following formula was used: cell viability $=(\mathrm{OD}$ of the experimental sample/ OD of the control group) $\times 100 \%$. 


\section{Analysis of cell death}

After treatment, cells were detached with trypsin, washed twice with $1 \times$ phosphate-buffered saline (PBS), and resuspended in annexin $\mathrm{V}$ binding buffer (7SeaPharmTech, Shanghai, China) at a concentration of $3 \times 10^{5}$ cells $/ \mathrm{ml}$. Next, the cells were incubated with fluorescein isothiocyanate-annexin $\mathrm{V}$ (7SeaPharmTech) for 15 minutes at room temperature in the dark and with propidium iodide (7SeaPharmTech) for 5 minutes at $4{ }^{\circ} \mathrm{C}$ in the dark, and then the cells were analyzed by flow cytometry (guava easyCyte HT; EMD Millipore, Billerica, MA, USA).

\section{Western blot analysis}

Synovial fibroblasts and tissue specimens were washed twice with ice-cold PBS and solubilized in Triton X-100 lysis buffer $(50 \mathrm{mM}$ Tris- $\mathrm{HCl}, \mathrm{pH}$ 7.4, $150 \mathrm{mM} \mathrm{NaCl}$, $0.2 \mathrm{mM}$ ethylenediaminetetraacetic acid, $1 \%$ Triton X-100, $1 \%$ sodium deoxycholate, $0.1 \%$ sodium dodecyl sulfate [SDS]) and protease inhibitor cocktail (Beyotime, Shanghai, China) on ice, then quantified using the Lowry method. Cell lysate proteins $(40 \mu \mathrm{g})$ were separated by SDSpolyacrylamide gel electrophoresis and electrophoretically transferred to nitrocellulose membranes (Immobilon-P; EMD Millipore). The membranes were blocked in $5 \%$ skim milk in Tris-buffered saline with Tween 20 (TBST) at room temperature for $1 \mathrm{~h}$ and incubated overnight at $4{ }^{\circ} \mathrm{C}$ with the indicated primary antibodies. After a washing step with TBST buffer, the membranes were reacted with the appropriate horseradish peroxidase-conjugated secondary antibodies for $1 \mathrm{~h}$ at room temperature. After incubation with the secondary antibody, the membranes were washed three times with TBST and developed via electrochemiluminescence (Thermo Fisher Scientific) and using a Western blotting detection system (GeneGnome 5; Syngene, Cambridge, UK).

\section{Transmission electron microscopy}

After treatment, cells were detached with trypsin, washed twice with PBS, and fixed in ice-cold $2 \%$ glutaraldehyde/0.1 M phosphate buffer ( $\mathrm{pH}$ 7.2), postfixed in $1 \%$ osmium tetroxide, washed, dehydrated with a graded ethanol series ( $30 \%, 50 \%, 70 \%, 90 \%$, and $100 \%)$, and embedded in 1:1 propylene oxide/embedding resin. The resin blocks were cut with a LKB V ultramicrotome (LKB, Bromma, Sweden). Thin (60-nm) sections were picked up on 200-mesh copper grids and stained with uranyl acetate and lead citrate. The sections were examined with an H-7650 transmission electron microscope (HITACHI, Ibaraki, Japan).

\section{Transfection experiments}

Both small interfering RNA (siRNA) targeting Beclin-1 complementary DNA (cDNA) sequence (5'-CAGTTTGG CACAATCAATATT-3') and HMGB1 cDNA sequence
(5'-CCCGTTATGAAAGAGAAATTT-3') and a control siRNA (5'-UUCUCCGAACGUGUCACGUTT-3') were obtained from Shanghai GenePharma (Shanghai, China). Cells were transfected with either Beclin-1 siRNA or control siRNA at $75 \mathrm{nmol} / \mathrm{L}$ using X-tremeGENE siRNA transfection reagent (Roche Diagnostics, Mannheim, Germany) according to the manufacturer's guidelines. Twenty-four hours after transfection, the cells were treated as indicated and then harvested for Western blot analysis or flow cytometry.

\section{Statistical analysis}

Mean \pm SD values were calculated. According to whether data are normally distributed, either the Mann-Whitney $U$ or Student's $t$ test was used for statistical evaluation of the data (GraphPad Prism 5.0 software; GraphPad Software, La Jolla, CA, USA). $p$ Values less than 0.05 were considered significant.

\section{Results \\ MTX inhibited cell viability and induced apoptosis}

RA-FLS and osteoarthritis fibroblast-like synovial cells (OA-FLS) were treated with MTX at concentrations ranging from $0.01 \mu \mathrm{M}$ to $10 \mu \mathrm{M}$ for $48 \mathrm{~h}$. By flow cytometry, assayed the number of dead cells after treatment with MTX, which showed that RA-FLS were more resistant than OA-FLS to MTX-induced cell death (Fig. 1a and b). Furthermore, cell viability assays showed that MTX inhibited cell growth in a dose-dependent manner (Fig. 1c). However, cell viability was $91.1 \pm 2.5 \%$ even when treated with MTX at a concentration of $1 \mu \mathrm{M}$ in RA-FLS, in contrast to $70.2 \pm 8.2 \%$ in OA-FLS.

\section{MTX induced autophagosome formation}

Both RA-FLS and OA-FLS were treated with MTX at concentrations ranging from $0.01 \mu \mathrm{M}$ to $10 \mu \mathrm{M}$ for $48 \mathrm{~h}$ and at times ranging from $12 \mathrm{~h}$ to $96 \mathrm{~h}$ at a concentration of $0.1 \mu \mathrm{M}$. An increase in type II LC3 (LC3-II) was observed in both a dose-dependent a time-dependent manner after treatment with MTX (Fig. 2a and b), indicating the induction of autophagy. In addition, the induction of autophagy was more pronounced in RA-FLS than in OA-FLS (Fig. 2a and b).

The increase in LC3-II, which is indicative of autophagy induction, reflects only the number of autophagosomes formed; it provides no information about the overall autophagic flux. Therefore, in this study, we stimulated RA-FLS with MTX in the presence and absence of the lysosomal inhibitor bafilomycin A1 to block the autophagic pathway at a late stage [20]. We showed that MTX treatment also increased the autophagic flux in RA-FLS, as demonstrated by an increased amount of LC3-II in the presence of bafilomycin A1 (Fig. 2c).We also detected the expression of P62 to confirm the 


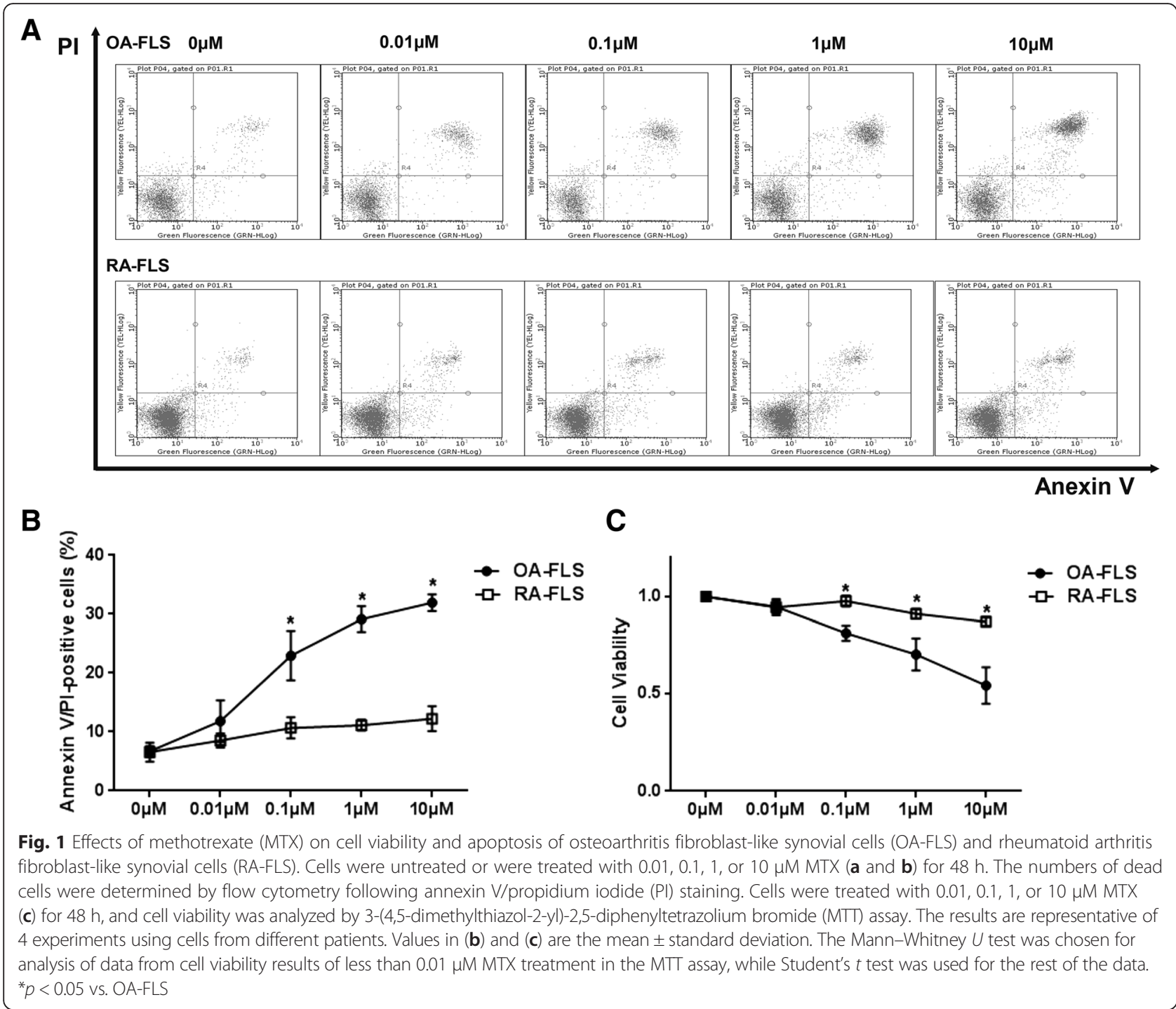

autophagic flux, but no significant changes were found (Additional file 1). The formation of autophagosomes was further confirmed by transmission electron microscopy. Upon treatment with $0.1 \mu \mathrm{M}$ MTX for $48 \mathrm{~h}$, many autophagic vesicles, double membrane-enclosed vesicles containing engulfed organelles, were observed in the cytoplasm of RA-FLS (Fig. 2d).

\section{MTX-induced autophagy protected RA-FLS from undergoing apoptosis}

Because autophagy can result in both survival and cell death in RA-FLS and OA-FLS, we next investigated whether MTX-induced autophagy is protective or proapoptotic. In this experiment, we examined the role of MTX-induced autophagy via knockdown of the autophagy marker Beclin-1. Figure 3a shows that the levels of Beclin-1 and LC3II were significantly decreased in Beclin-1 siRNA-treated cells compared with the results in siRNA controls. In RA-FLS, but not in OA-FLS, the Beclin-1 siRNA significantly increased the apoptotic population with $0.1 \mu \mathrm{M}$ MTX (Fig. 3b). To further confirm that apoptosis was induced by MTX, Western blotting was performed to detect the cleavage of PARP. As shown in Fig. 3c, after $0.1 \mu \mathrm{M}$ MTX treatment for $48 \mathrm{~h}$, the cleavage of PARP was increased more dramatically in the RA-FLS transferred with Beclin-1 siRNA than with the control siRNA, but it showed no comparable change in OA-FLS transferred with Beclin-1 siRNA.

\section{MTX induced autophagy through HMGB1 and Beclin-1, not the Akt/mTOR pathway}

To explore how MTX induces autophagy in RA-FLS and OA-FLS treated with MTX at times ranging from $12 \mathrm{~h}$ to $96 \mathrm{~h}$ at a concentration of $0.1 \mu \mathrm{M}$, two important autophagy-related signaling pathways were investigated: the Akt/mTOR signaling pathway and the HMGB1/ 


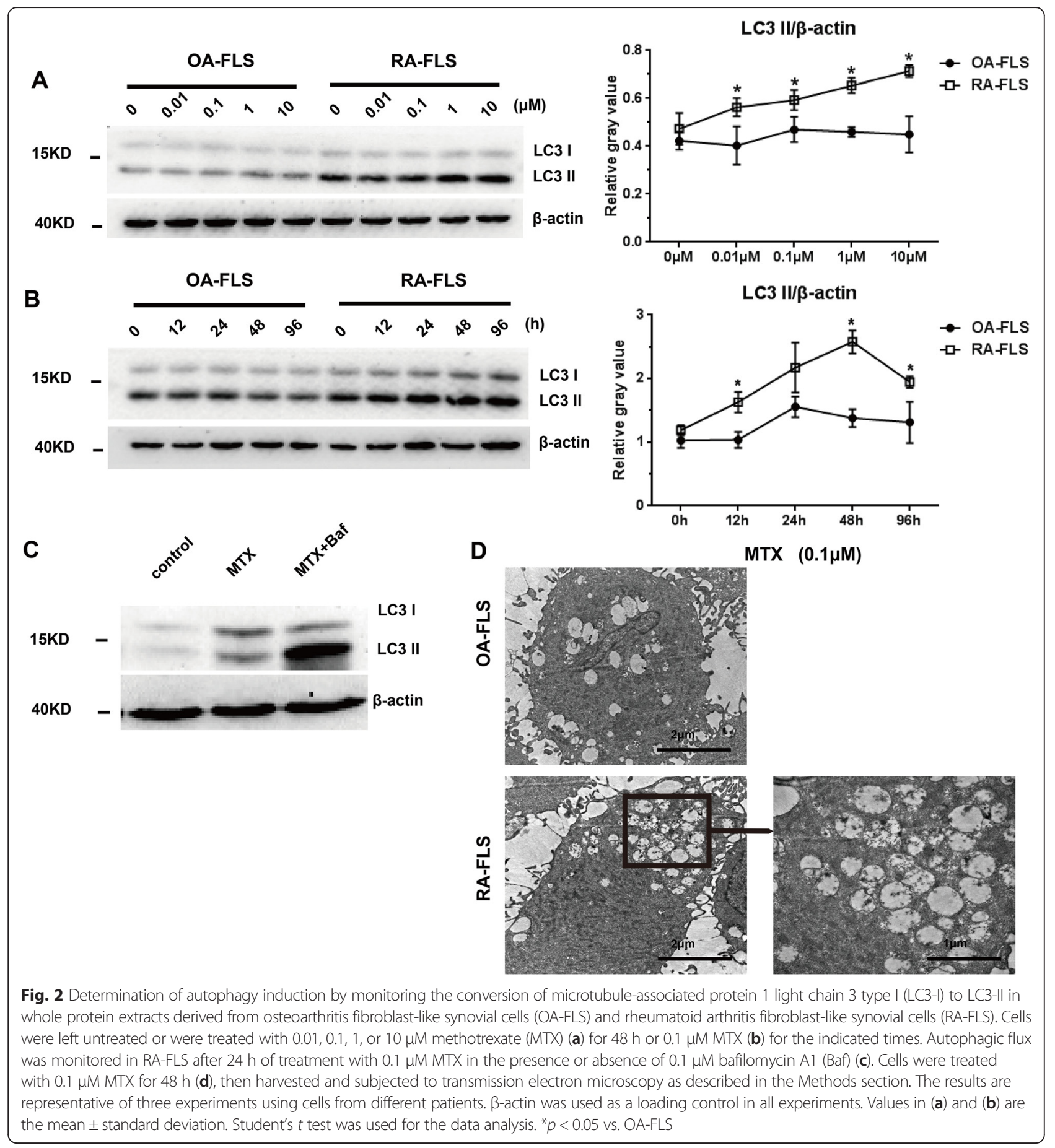

Beclin-1 pathway. As detected by Western blotting, the levels of phospho-Akt and downstream p-mTOR were more powerful in RA-FLS but were not affected by prolonging the duration of MTX in both RA-FLS and OA-FLS. Interestingly, the expression of HMGB1 and Beclin-1 was upregulated in a time-dependent manner in RA-FLS, but in OA-FLS it was very limited (Fig. 4a and $\mathrm{b}$ ). These data indicated that MTX-induced autophagy might occur via upregulated HMGB1 and Beclin-1 rather than through the Akt/mTOR signaling pathway in RA-FLS.

\section{Autophagy induction contributed to resistance to MTX treatment in RA-FLS through HMGB1}

We further confirmed the role of HMGB1 in MTXinduced autophagy via knockdown of the expression of 


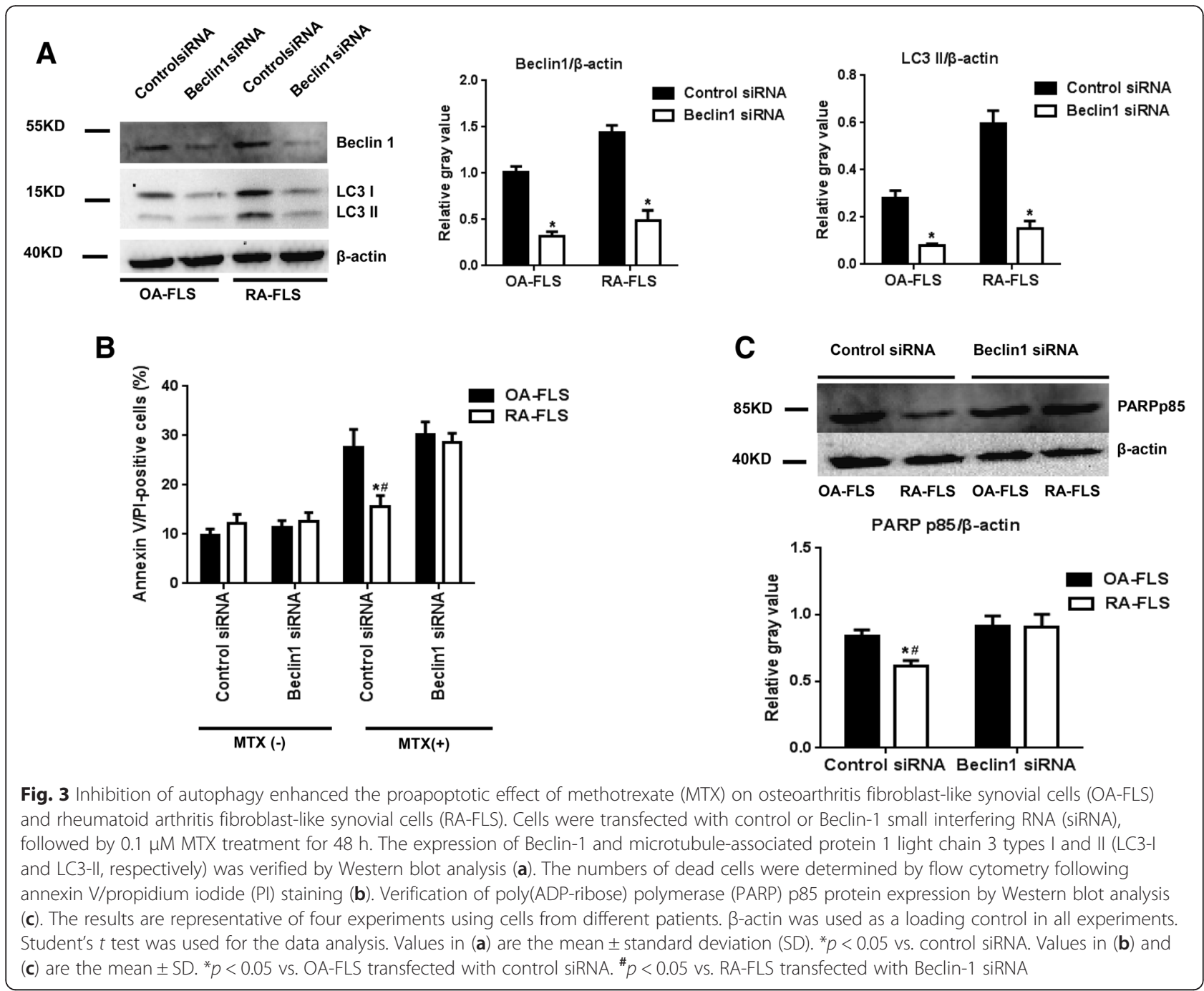

HMGB1. Figure $5 \mathrm{a}-\mathrm{c}$ shows that the protein levels of HMGB1 and LC3-II were significantly decreased in HMGB1 siRNA-treated cells compared with the results with the siRNA controls. In RA-FLS, the HMGB1 siRNA significantly increased the apoptotic population compared with the siRNA controls (Fig. 5d and e).

\section{Discussion}

A substantial increase in the number of RA-FLS, partly but importantly resulting in synovial hyperplasia, is partially caused by resistant apoptosis. Different from either nonarthritic controls or patients with OA, RA-FLS hardly show any signs of apoptosis, which was also observed in animal model studies. Furthermore, RA-FLS also exhibited resistance to apoptotic stimulation in a number of in vitro studies. However, proliferation of RA-FLS seems to contribute to synovial hyperplasia. It has been reported in several research articles that some cellular proliferation regulators, such as Ras and c-Myc, are overexpressed in RA and that the inhibition of these regulators can reduce the growth of RA-FLS both in vitro and in vivo $[21,22]$.

One of the unique effects of MTX is the induction of RA-FLS apoptosis, compared with other DMARDs, which was first described by Nakazawa et al. [10]. Nevertheless, this effect was later shown to be compromised. Terminal deoxynucleotidyl transferase deoxyuridine triphosphate nick end labeling- and PARP-positive cells were found mainly in the synovial lining macrophages, but not in RA-FLS or other cell types, from RA synovial biopsy specimens after MTX treatment [11]. Lee et al. demonstrated apoptosis of RA-FLS treated with 100$500 \mu \mathrm{M}$ MTX in cell culture [9], which is a concentration that cannot be achieved in the plasma. In the present study, we demonstrated a dose-effect relationship between MTX and apoptosis of RA-FLS and OA-FLS. However, apoptotic cells were detectable in the RA-FLS $(11.1 \pm 0.9 \%)$ cultured with MTX at a high dose of up to 
A

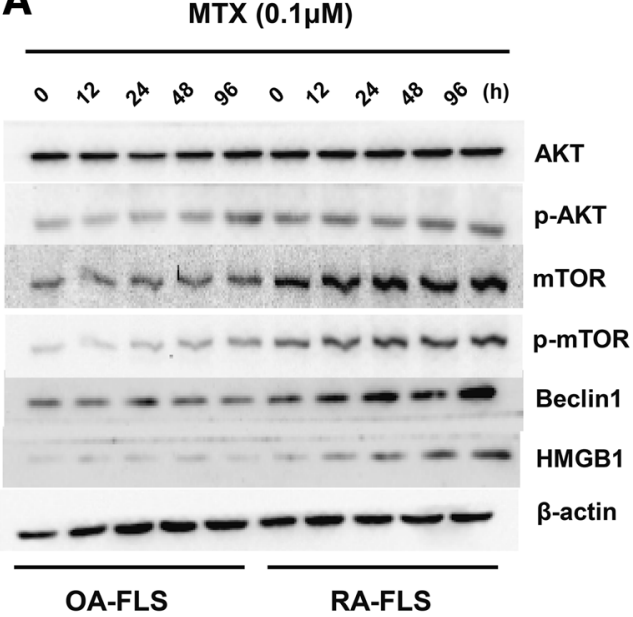

B
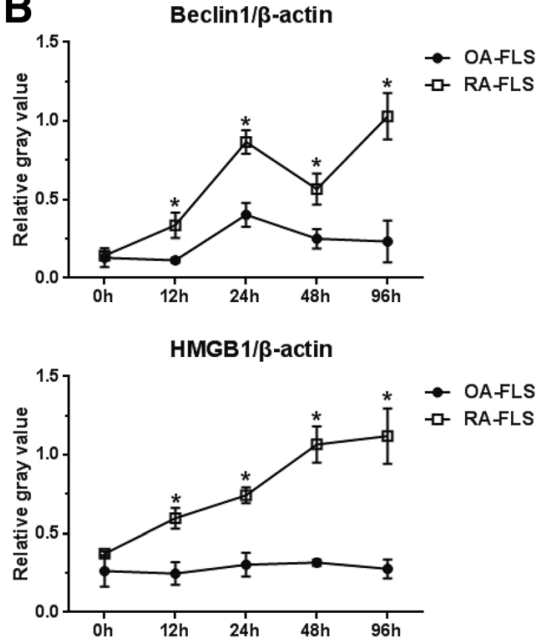

Fig. 4 Effect of methotrexate (MTX) on autophagy-related proteins. a After the cells were exposed to $0.1 \mu \mathrm{M}$ MTX for the indicated times, the cell lysates were subjected to Western blotting with specific antibodies. The results are representative of three independent experiments. $\beta$-actin was used as a loading control. b The levels of Akt, phosphorylated Akt (p-Akt), mammalian target of rapamycin (mTOR), phosphorylated mTOR (p-mTOR), high mobility group box chromosomal protein 1 (HMGB1), Beclin-1, and microtubule-associated protein 1 light chain 3 (LC3) proteins were measured using ImageJ software (National Institutes of Health, Bethesda, MD, USA) and corrected for $\beta$-actin. Data in columns represent mean of three independent experiments, and values in (b) are the mean \pm standard deviation. The Mann-Whitney $U$ test was chosen for analysis of data for the levels of HMGB1 of cells exposed to $0.1 \mu \mathrm{M}$ MTX for $48 \mathrm{~h}$ in Western blot assays, while Student's $t$ test was used for the rest of the data. ${ }^{*} p<0.05$ vs. OA-FLS

$1 \mu \mathrm{M}$, significantly less than OA-FLS $(29.2 \pm 2.2 \%)$. In contrast, pharmacokinetic analysis indicates that the ingestion of a 20-mg tablet of MTX yields plasma MTX concentrations of approximately $0.5 \mu \mathrm{M}$ after $1 \mathrm{~h}$ and approximately $0.1 \mu \mathrm{M}$ after $10 \mathrm{~h}$.

Apart from the capacity of RA-FLS for apoptotic resistance, advanced sensitivity to induced autophagy seems to be another characteristic of RA-FLS that is in contrast to OA-FLS $[11,16,17,23]$; however, it is rarely reported whether MTX has the ability to induce autophagy in RA-FLS. In our study, the increased expression of punctate LC3 and the morphologic changes were observed among the cells treated with MTX. Western blot analysis also showed that LC3-II expression was elevated with MTX treatment in a dose-dependent and timedependent manner. If the increase in both LC3-II and autophagosome occurs only as a result of either upregulation of autophagosome formation or blockage of autophagic degradation, autophagic flux would be detected [24]. We found that in RA-FLS treated with $0.1 \mu \mathrm{M}$ MTX, LC3-II further accumulated in the presence of bafilomycin A1, a lysosomal protease inhibitor. This finding indicates enhancement of autophagic flux and upregulated autophagosome formation by MTX [20]. To our knowledge, this is the first report that MTX induces autophagy in RA-FLS.

Autophagy has been shown to engage in a complex interplay with cell survival. Sometimes it induces an apoptotic or autophagic cell death accompanied by massive cytoplasmic vacuolization; at other times, it serves as a protector, as observed in RA-FLS. The studies with patients with RA by Shin et al. demonstrated that autophagy protects cells from death by limiting the endoplasmic reticulum stress response in fibroblasts [16]. However, a more recent study indicated a different result. Autophagy induced by different stimuli may lead to different and even opposite consequences. Autophagy seems to play a dual role in the survival of RA-FLS [23]. To clarify the consequences of autophagy induced by MTX, we inhibited autophagic activity by transferring Beclin-1 siRNA in RA-FLS, which significantly decreased the Beclin-1 expression and subsequently resulted in increased apoptosis with MTX treatment. Under our experimental conditions, MTXinduced autophagy served as a protector in RA-FLS.

The mTOR-dependent pathway is a classical regulator of autophagy [25]. The target of rapamycin (TOR) kinase is activated downstream of Akt kinase, phosphoinositide 3-kinase (PI3K), and growth factor receptor, signaling when nutrients are available. Upstream of TOR, the activation of adenosine 5'-monophosphate-activated protein kinase in response to low ATP levels promotes inhibitory activity of the Tsc1/Tsc2 tumor suppressor proteins on Rheb, a small GTPase required for mTOR activity. Reduced Akt activity in response to reduced growth factor receptor activity also represses TOR kinase through Tsc1 and Tsc2 [26, 27]. Thus, reduced TOR activity induces autophagy. In this study, the expressions of p-Akt and p-mTOR were higher in RA-FLS than in OA-FLS, but these seemed irrelevant to the use of MTX. 


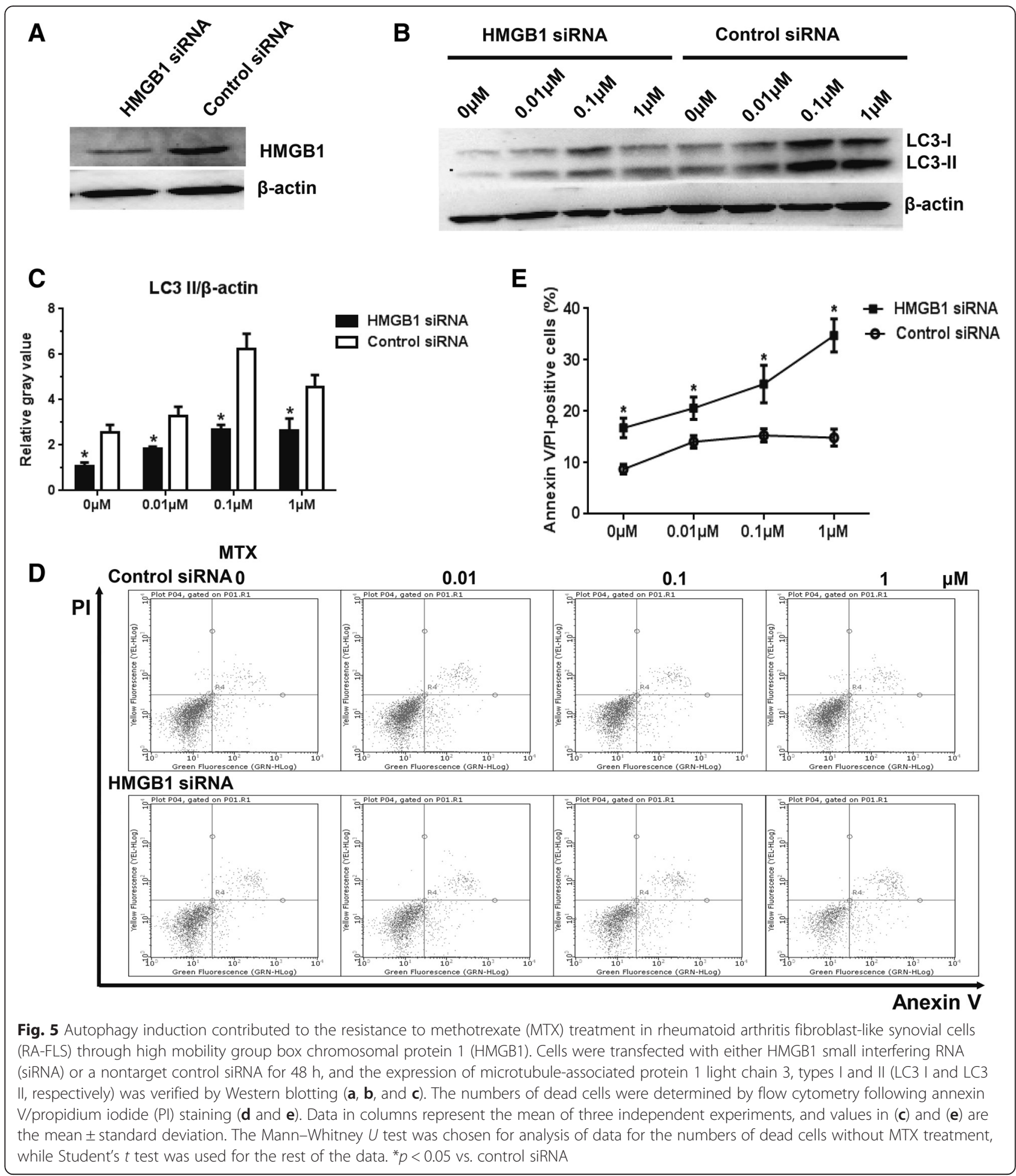

Autophagy induced under pathological conditions functions as an adaptive cell response that allows the cell to survive bioenergetic stress [28]. However, extensive or persistent autophagy also results in cell death [29]. Bielen et al. found that phosphorylation of plateletderived growth factor receptor (PDGFR) $\alpha / \beta$ suppresses autophagy by activating the PI3K/Akt/mTOR signaling pathway, thereby preventing RA-FLS from undergoing type II apoptosis induced by excessive autophagy and leading to continuing proliferation of RA-FLS and aggravation of the pathogenetic condition [30]. The activation of the PI3K/Akt/mTOR signaling pathway may prevent 
RA-FLS from excessive autophagy and avoid type II apoptosis. Therefore, this may be another survival mechanism of drug treatment resistance. Nevertheless, why autophagy is robust in RA-FLS after MTX treatment remains unknown.

Fortunately, MTX induced in a time-dependent manner the expression of Beclin-1 and HMGB1 in RA-FLS, but this was very limited in OA-FLS. Beclin1 , the mammalian homolog of the yeast Atg6, is a key autophagy-promoting gene whose product is part of a lipid kinase (class III PI3K [PtdIns3KC3]) complex that participates in the early stages of autophagosome formation [31]. Upregulated HMGB1 competes with Bcl-2 to bind Beclin-1, which increases the formation of the Beclin-1-PtdIns3KC3 complex and stimulates autophagosome maturation and autophagy. As an upstream signal, the activation of the ULK1mAtg13-FIP200 complex is required for the interaction between HMGB1 and Beclin-1 [32, 33]. We also demonstrated that autophagy activity was downregulated after transferring HMGB1 siRNA. Therefore, the robust autophagy of RA-FLS with MTX treatment seems to be caused by HMGB1.

In addition, we found that the inhibition of HMGB1 increased the apoptotic population of RA-FLS after MTX treatment. HMGB1 is an inducer of autophagy. Our study showed that knockdown of HMGB1 decreased LC3-II levels and inhibited autophagy activity, resulting in increased apoptosis, which is the same result found in non-small cell lung cancer [34]. Apart from its proautophagic effect and its interactions with receptor for advanced glycation endproducts (RAGE) and Tolllike receptor 4, HMGB1 plays a crucial role in various inflammation processes [35]. The induction of HMGB1 may mediate proinflammatory action in RA-FLS, while inhibition of the HMGB1-RAGE interaction may have anti-inflammatory effects in RA [36, 37]. The inhibition of the proinflammatory effects through the transfer of HMGB1 siRNA may be another cause of the increased drug sensitivity of RA-FLS. However, further study is required to explain how MTX induces the expression of HMGB1 and Beclin-1.

\section{Conclusions}

The results of the present study suggest that RA-FLS may use the autophagic pathway in which HMGB1 and Beclin-1 (but not the Akt/mTOR pathway) are involved as a survival mechanism to evade the perturbations of cellular biosynthetic processes by the antimetabolite MTX to sustain cell viability in conditions of metabolic stress. Thus, a combination of MTX and an autophagy inhibitor might be more effective for the treatment of RA.

\section{Additional file}

Additional file 1: The expression of P62 in RA-FLS and OA-FLS treated with $0.1 \mu \mathrm{M}$ MTX for the indicated times (TIF $698 \mathrm{~kb}$ )

\section{Abbreviations}

Baf: bafilomycin A1; CCP: cyclic citrullinated peptide; cDNA: complementary DNA; CRP: C-reactive protein; DMARD: disease-modifying antirheumatic drug; DMSO: dimethyl sulfoxide; ESR: erythrocyte sedimentation rate; FLS: fibroblastlike synovial cells; HMGB1: high mobility group box chromosomal protein 1; LC3: microtubule-associated protein 1 light chain 3; LC3-II: microtubuleassociated protein 1 light chain 3, type Il; mTOR: mammalian target of rapamycin; MTT: 3-(4,5-dimethylthiazol-2-yl)-2,5-diphenyltetrazolium bromide; MTX: methotrexate; NSAID: nonsteroidal anti-inflammatory drug; OA: osteoarthritis; OA-FLS: osteoarthritis fibroblast-like synovial cells; OD: optical density; PBS: phosphate-buffered saline; PARP: poly(ADP-ribose) polymerase; PI: propidium iodide; PI3K: phosphoinositide 3-kinase; p-mTOR: phosphorylated mammalian target of rapamycin; Ptdlns3KC3: class III phosphoinositide 3-kinase; RA: rheumatoid arthritis; RA-FLS: rheumatoid arthritis fibroblast-like synovial cells; RAGE: receptor for advanced glycation endproducts; RF: rheumatoid factor; SD: standard deviation; SDS: sodium dodecyl sulfate; siRNA: small interfering RNA; TBST: Tris-buffered saline with Tween 20; TOR: target of rapamycin; TWP: Tripterygium wilfordii polyglycoside.

\section{Competing interests}

The authors declare that they have no competing interests.

\section{Authors' contributions}

All authors were involved in drafting the manuscript. PX and S-ML conceived of the study, carried out the experiments, and analyzed the data. KX and YC carried out the experiments, collected the clinical patients, performed the statistical analysis, and participated in the study design. $X L$ and $L L$ collected the clinical patients and participated in the study design. ZL and HL participated in the interpretation of the data. All authors read and approved the final manuscript.

\section{Acknowledgments}

The authors are grateful for the technical advice and collection of specimens provided by Jing Wang and Congshan Jiang. This work was supported by the National Natural Science Foundation of China (grants 81271948 and 81171742).

\section{Author details}

${ }^{1}$ Department of Joint Surgery, Xi'an Hong Hui Hospital, Xi'an Jiaotong University Health Science Center, Xi'an 710054, Shaanxi Province, China. ${ }^{2}$ Department of Genetics and Molecular Biology, Xi'an Jiaotong University Health Science Center, Xi'an, Shaanxi Province, China. ${ }^{3}$ Department of Internal Medicine, Xi'an Hong Hui Hospital, Xi'an Jiaotong University Health Science Center, Xi'an, Shaanxi Province, China.

Received: 1 October 2015 Accepted: 8 December 2015

Published online: 23 December 2015

\section{References}

1. Leech MT, Morand EF. Fibroblasts and synovial immunity. Curr Opin Pharmacol. 2013;13(4):565-9.

2. Meinecke I, Rutkauskaite E, Gay S, Pap T. The role of synovial fibroblasts in mediating joint destruction in rheumatoid arthritis. Curr Pharm Des. 2005;11(5):563-8.

3. Cronstein BN. Low-dose methotrexate: a mainstay in the treatment of rheumatoid arthritis. Pharmacol Rev. 2005;57(2):163-72.

4. Weinblatt ME, Coblyn JS, Fox DA, Fraser PA, Holdsworth DE, Glass DN, et al. Efficacy of low-dose methotrexate in rheumatoid arthritis. N Engl J Med. 1985;312(13):818-22.

5. Phillips DC, Woollard KJ, Griffiths HR. The anti-inflammatory actions of methotrexate are critically dependent upon the production of reactive oxygen species. Br J Pharmacol. 2003;138(3):501-11.

6. Spurlock CF, Tossberg JT, Fuchs HA, Olsen NJ, Aune TM. Methotrexate increases expression of cell cycle checkpoint genes via JNK activation. Arthritis Rheum. 2012;64(6):1780-9. 
7. Sung JY, Hong JH, Kang HS, Choi I, Lim SD, Lee JK, et al. Methotrexate suppresses the interleukin-6 induced generation of reactive oxygen species in the synoviocytes of rheumatoid arthritis. Immunopharmacology. 2000:47(1):35-44.

8. Kammouni W, Wong K, Ma G, Firestein GS, Gibson SB, El-Gabalawy HS Regulation of apoptosis in fibroblast-like synoviocytes by the hypoxia-induced Bcl-2 family member Bcl-2/adenovirus E1B 19-kd protein-interacting protein 3. Arthritis Rheum. 2007;56(9):2854-63.

9. Lee SY, Park SH, Lee SW, Lee SH, Son MK, Choi YH, et al. Synoviocyte apoptosis may differentiate responder and non-responder patients to methotrexate treatment in rheumatoid arthritis. Arch Pharm Res. 2014;37(10):1286-94

10. Nakazawa F, Matsuno H, Yudoh K, Katayama R, Sawai T, Uzuki M, et al. Methotrexate inhibits rheumatoid synovitis by inducing apoptosis. J Rheumatol. 2001;28(8):1800-8.

11. Smith MD, Weedon H, Papangelis V, Walker J, Roberts-Thomson PJ, Ahern MJ. Apoptosis in the rheumatoid arthritis synovial membrane: modulation by disease-modifying anti-rheumatic drug treatment. Rheumatology (Oxford). 2010:49(5):862-75.

12. Liu J, Zhang Y, Qu J, Xu L, Hou K, Zhang J, et al. $\beta$-Elemene-induced autophagy protects human gastric cancer cells from undergoing apoptosis. BMC Cancer. 2011;11:183

13. Sato K, Tsuchihara K, Fujii S, Sugiyama M, Goya T, Atomi Y, et al. Autophagy is activated in colorectal cancer cells and contributes to the tolerance to nutrient deprivation. Cancer Res. 2007;67(20):9677-84.

14. Zhang J, Li Y, Chen X, Liu T, Chen Y, He W, et al. Autophagy is involved in anticancer effects of matrine on SGC-7901 human gastric cancer cells. Oncol Rep. 2011;26(1):115-24.

15. Allan LA, Clarke PR. Apoptosis and autophagy: regulation of caspase- 9 by phosphorylation. FEBS J. 2009;276(21):6063-73.

16. Shin YJ, Han SH, Kim DS, Lee GH, Yoo WH, Kang YM, et al. Autophagy induction and CHOP under-expression promotes survival of fibroblasts from rheumatoid arthritis patients under endoplasmic reticulum stress. Arthritis Res Ther. 2010;12(1):R19.

17. Xu K, Xu P, Yao JF, Zhang YG, Hou WK, Lu SM. Reduced apoptosis correlates with enhanced autophagy in synovial tissues of rheumatoid arthritis. Inflamm Res. 2013;62(2):229-37.

18. Altman R, Asch E, Bloch D, Bole G, Borenstein D, Brandt K, et al. Development of criteria for the classification and reporting of osteoarthritis: classification of osteoarthritis of the knee. Arthritis Rheum. 1986;29(8):1039-49.

19. Combe B, Edno L, Lafforgue P, Bologna C, Bernard JC, Acquaviva P, et al. Total and free methotrexate pharmacokinetics, with and without piroxicam, in rheumatoid arthritis patients. Br J Rheumatol. 1995;34(5):421-8.

20. Mizushima N, Yoshimori T. How to interpret LC3 immunoblotting. Autophagy. 2007;3(6):542-5.

21. Qu Z, Garcia CH, O'Rourke LM, Planck SR, Kohli M, Rosenbaum JT. Local proliferation of fibroblast-like synoviocytes contributes to synovial hyperplasia. Arthritis Rheum. 1994;37(2):212-20.

22. Pap $T$, Nawrath $M$, Heinrich J, Bosse M, Baier A, Hummel KM, et al. Cooperation of Ras- and C-Myc-dependent pathways in regulating the growth and invasiveness of synovial fibroblasts in rheumatoid arthritis Arthritis Rheum. 2004;50(9):2794-802.

23. Kato M, Ospelt C, Gay RE, Gay S, Klein K. Dual role of autophagy in stress-induced cell death in rheumatoid arthritis synovial fibroblasts. Arthritis Rheumatol. 2014;66(1):40-8.

24. Mizushima N, Yoshimori T, Levine B. Methods in mammalian autophagy research. Cell. 2010;140(3):313-26.

25. Lum JJ, Bauer DE, Kong M, Harris MH, Li C, Lindsten T, et al. Growth factor regulation of autophagy and cell survival in the absence of apoptosis. Cell. 2005;120(2):237-48.

26. Garami A, Zwartkruis FJT, Nobukuni T, Joaquin M, Roccio M, Stocker H, et al. Insulin activation of Rheb, a mediator of mTOR/S6K/4E-BP signaling, is inhibited by TSC1 and 2. Mol Cell. 2003;11(6):1457-66.

27. Inoki K, Li Y, Zhu TQ, Wu J, Guan KL. TSC2 is phosphorylated and inhibited by Akt and suppresses mTOR signalling. Nat Cell Biol. 2002;4(9):648-57.

28. Levine B. Cell biology: autophagy and cancer. Nature. 2007:446(7137):745-7.

29. Levine B, Yuan J. Autophagy in cell death: an innocent convict? J Clin Invest. 2005:115(10):2679-88.

30. Bielen A, Perryman L, Box GM, Valenti M, de Haven BA, Martins V, et al. Enhanced efficacy of IGF1R inhibition in pediatric glioblastoma by combinatorial targeting of PDGFRa/ $\beta$. Mol Cancer Ther. 2011;10(8):1407-18.
31. Tian S, Lin J, Zhou J, Wang X, Li Y, Ren X, et al. Beclin 1-independent autophagy induced by a $\mathrm{BCl}-\mathrm{X}_{\perp} / \mathrm{BCl}-2$ targeting compound, Z18. Autophagy. 2010;6(8):1032-41.

32. Huang J, Ni J, Liu K, Yu Y, Xie M, Kang R, et al. HMGB1 promotes drug resistance in osteosarcoma. Cancer Res. 2012;72(1):230-8.

33. Guo S, Bai R, Liu W, Zhao A, Zhao Z, Wang Y, et al. miR-22 inhibits osteosarcoma cell proliferation and migration by targeting HMGB1 and inhibiting HMGB1-mediated autophagy. Tumour Biol. 2014;35(7):7025-34.

34. Zhang R, Li Y, Wang Z, Chen L, Dong X, Nie X. Interference with HMGB1 increases the sensitivity to chemotherapy drugs by inhibiting HMGB1-mediated cell autophagy and inducing cell apoptosis. Tumour Biol. 2015;36(11):8585-92.

35. Andersson $\mathrm{U}$, Tracey KJ. HMGB1 is a therapeutic target for sterile inflammation and infection. Annu Rev Immunol. 2011:29:139-62.

36. Qin Y, Chen Y, Wang W, Wang Z, Tang G, Zhang P, et al. HMGB1-LPS complex promotes transformation of osteoarthritis synovial fibroblasts to a rheumatoid arthritis synovial fibroblast-like phenotype. Cell Death Dis. 2014;5:e1077.

37. Kuroiwa Y, Takakusagi Y, Kusayanagi T, Kuramochi K, Imai T, Hirayama T, et al. Identification and characterization of the direct interaction between methotrexate (MTX) and high-mobility group box 1 (HMGB1) protein. PLoS One. 2013;8(5):e63073.

\section{Submit your next manuscript to BioMed Central and we will help you at every step:}

- We accept pre-submission inquiries

- Our selector tool helps you to find the most relevant journal

- We provide round the clock customer support

- Convenient online submission

- Thorough peer review

- Inclusion in PubMed and all major indexing services

- Maximum visibility for your research

Submit your manuscript at www.biomedcentral.com/submit
) Biomed Central 